\title{
A MEASURE-THEORETIC PROOF OF THE STONE-WEIERSTRASS APPROXIMATION THEOREM
}

\author{
SURJTT SINGH KHURANA
}

\begin{abstract}
Using the Daniell integral, a simple proof of the Stone-Weierstrass
\end{abstract} theorem is obtained.

A simple measure-theoretic proof of the following version of the StoneWeierstrass approximation theorem is given.

THEOREM. Let $X$ be a compact Hausdorff space, $C(X)$ all continuous real-valued functions on $X$, and $L$ a point-separating linear sublattice of $C(X)$ such that $1 \in L$. Then $L$ is norm dense in $C(X)$.

Proof. Let $\mu$ be a positive regular Borel measure on $X . \mu_{\mid L}$ is a Daniell integral [2, p. 287] and as such can be uniquely extended to a Daniell integral $\mu: L_{1} \rightarrow R$ with the property that $L_{1}$ contains the characteristic functions of a $\sigma$-algebra $\mathfrak{A}$ such that each function in $L_{1}$ is $\mathfrak{A}$-measurable [2, Chapter 13]. Since $L$ separates points of $X$ and elements of $L$ are $\mathfrak{X}$-measurable, open subsets of $X$ in $\mathfrak{A}$ form a base for a Hausdorff topology on $X$ and as such form a base of the original topology on $X$. Now take a $\mu \in(C(X))^{\prime}, \mu \equiv 0$ on $L$. By taking its positive and negative parts, $\mu$ can be considered as a Daniell integral on both $A=C(X)$ and on $L$. By the uniqueness of extension [2, Proposition 14] if $\mu$ is the extension of $\mu$ to $A_{1}$ and $\mu_{1}$ is the extension of $\mu$ to $L_{1}$, then $A_{1} \supset L_{1}, \mu=\mu_{1}$ on $L_{1}$, and $\mu \equiv 0$ on $L_{1}$. Thus $\mu \equiv 0$ on a certain base of open subsets of $X$. By the regularity of $\mu, \mu \equiv 0$ on all open subsets of $X$ and as such $\mu \equiv 0$. (Note if $\left\{V_{\alpha}\right\}$ is an increasing net of open sets with $V=\cup V_{\alpha}$ then for any positive regular Borel measure $\nu$ on $X, \nu(V)=$ $\lim \nu\left(V_{\alpha}\right)$, for if a compact $C \subset V$, then $C \subset V_{\alpha}$ for some $\alpha$; from this it is immediate that for any signed measure $\mu, \mu(V)=\lim \mu\left(V_{\alpha}\right)$.) By the Hahn-Banach theorem, $L$ is norm dense in $C(X)$.

REMARK. If $L$ is a point-separating subalgebra of $C(X)$ with $1 \in L$, then it is easy to prove that its closure $\bar{L}$ is a lattice [1] and so $L$ is norm dense in $C(X)$.

\section{REFERENCES}

1. R. G. Douglas, On lattices and algebras of real-valued functions, Amer. Math. Monthly 72 (1965), 642-643.

2. L. Royden, Real analysis, Macmillan, New York, 1968.

Departmant of Mathematics, University of Iowa, Iowa Ctry, Iowa 52242

Received by the editors June 13, 1979 and, in revised form, August 7, 1979.

AMS (MOS) subject classifications (1970). Primary 46B25, 46E05, 46E15.

Key words and phrases. Regular Borel measures, Daniell integral, Hahn-Banach theorem. 